\section{Evaluación de Tecnología Sanitaria y su impacto en políticas de tarificación y cobertura de medicamentos}

\author{
CARLA CASTILLO-LABORDE, ${ }^{1, a}$ NICOLÁS SILVA-ILLANES²,b
}

\section{Health technology assessment and its impact on pharmaceutical pricing and reimbursement policies}

The article conceptualizes the pharmaceutical pricing and reimbursement policies related to financial coverage in the context of health systems. It introduces the pharmaceutical market as an imperfect one, in which appropriate regulation is required. Moreover, the basis that guide the pricing and reimbursement processes are defined and described in order to generate a categorization based on whether they are intended to assess the 'added value' and if the evaluation is based on cost-effectiveness criteria. This framework is used to review different types of these policies applied in the international context, discussing the role of the Health Technology Assessment in these processes. Finally, it briefly discusses the potential role of these types of policies in the Chilean context.

(Rev Med Chile 2014; 142 (S1): S 33-38)

Key words: Pharmaceutical Policy; Pricing; Reimbursement, Health Insurance; Technology Assessment, Biomedical.

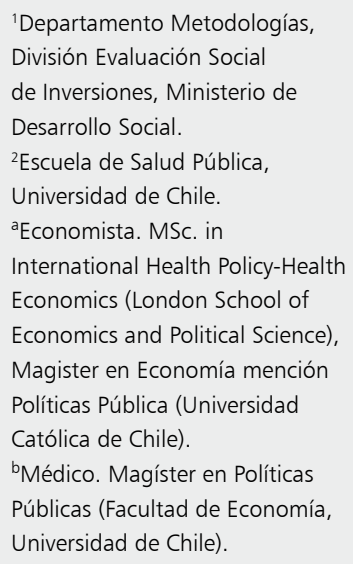

Este artículo no contó con ningún tipo de apoyo financiero. Los autores declaran no tener conflictos de intereses.

Este artículo fue sometido a revisión por pares.

Correspondencia a: Carla Castillo-Laborde Dirección: Ahumada 48, Piso 6 , Santiago Centro. ccastillol@desarrollosocial.c
E mercado de los medicamentos es uno particular, alejado de lo que se considera un mercado en competencia perfecta y por esto, uno de los mercados más regulados. Debido a que la producción de fármacos requiere realizar inversiones importantes antes de su lanzamiento al mercado y que a su vez los costos de manufactura son muy bajos en comparación con los asociados al desarrollo de estos productos, existe un sistema de patentes orientado a proteger la innovación y desarrollo de nuevas terapias, y de esta forma corregir esta falla de mercado. En base a esto es posible distinguir entre aquellos medicamentos con patente vigente y aquellos sin patente, estos últimos a su vez se pueden diferenciar entre aquellos de marca pero con patente vencida y los genéricos, que también pueden tener marca o sólo identificarse por su principio activo.

En el caso de los medicamentos con patente vigente, el mercado funciona de manera cercana a un monopolio o competencia monopolística, debido a la aparición de medicamentos sólo ligeramente diferentes que también pueden contar con patente. Por otra parte, una vez que la patente expira, al removerse la barrera de entrada legal, se esperaría que una mayor competencia tomara lugar en el mercado de este medicamento ${ }^{1}$, lo cual no siempre se produce. Estas y otras características propias de este mercado han hecho que se diseñen diversos tipos de políticas públicas regulatorias con el objetivo de lograr un equilibrio entre alcanzar equidad, eficiencia, satisfacer las necesidades de los pacientes y también balancear los objetivos de las políticas de salud con aquellos de la industria ${ }^{2}$.

El objetivo del presente artículo es revisar uno de estos tipos de políticas de regulación denominadas en forma general "políticas de tarificación y reembolso". Para ello se realiza una descripción general de los objetivos de este conjunto de políticas, se exponen de manera sintética sus características, se revisan experiencias internacionales destacadas y se discute el rol de la Evaluación de 
Tecnologías Sanitarias (ETESA) en este tipo de regulación.

\section{Políticas de tarificación y reembolso}

La regulación de precios tiene como objetivo asegurar que el precio pagado por los medicamentos (ya sea por fondos públicos o por los pacientes) no supere un valor "justo", en un contexto de salud pública, a la luz de principios como la solidaridad y la eficiencia, y que sea capaz de balancear los objetivos de la sociedad y de la industria ${ }^{3}$. Se tienen entonces consideraciones de accesibilidad financiera, contención del gasto, disponibilidad de nuevas terapias, importancia de la industria farmacéutica para la economía nacional, entre otras.

Por otra parte, el nivel de reembolso o cobertura financiera, y por lo tanto el cargo a los usuarios, es el resultado de la negociación entre la compañía farmacéutica (generalmente monopolistas en el caso de productos nuevos) y el pagador (gobierno, seguro público, seguro privado). En este caso se busca afectar el nivel de precio y la utilización, entregando señales a la industria sobre cuál es el nivel de precios aceptable a pagar por una determinada ganancia clínica, debido a esto las políticas de reembolso se relacionan más con la eficiencia económica que las de fijación de precio ${ }^{4}$. Se debe definir a qué productos otorgar cobertura y, cuánta cobertura otorgarles.
De manera general, el contexto en el que se desarrollan estas políticas es en el de países con sistemas de salud integrados y con cobertura universal, en donde el aseguramiento incluye medicamentos ambulatorios y hospitalizados. En estos países las decisiones de cobertura, tarificación y reembolso se llevan a cabo de forma centralizada. La Figura 1 entrega un esquema que representa como se llevan a cabo estos procesos.

En primer lugar la ETESA permite entregar información a los tomadores de decisión con respecto a si otorgar o no cobertura a un determinado tratamiento. Los criterios con respecto a qué tratamientos se someten a evaluación varían entre países: mientras algunos evalúan todos los fármacos, otros establecen ciertos criterios para determinar aquellos que requieren una evaluación formal.

El resultado de la decisión con respecto a cobertura puede simplificarse en cuatro alternativas: otorgar cobertura, otorgar cobertura con ciertas restricciones (a un determinado grupo de pacientes o bajo condiciones específicas), otorgar cobertura sujeto a recolección de evidencia, o no otorgarla. Los países suelen llevar a cabo esta decisión mediante un sistema de "listas positivas" en las que se incluyen los tratamientos que serán cubiertos o mediante "listas negativas" en donde se señalan los tratamientos que no serán sujetos de cobertura ${ }^{5}$.
Figura 1. ETESA y proceso de cobertura, tarificación y reembolso. Fuente: Elaboración propia.

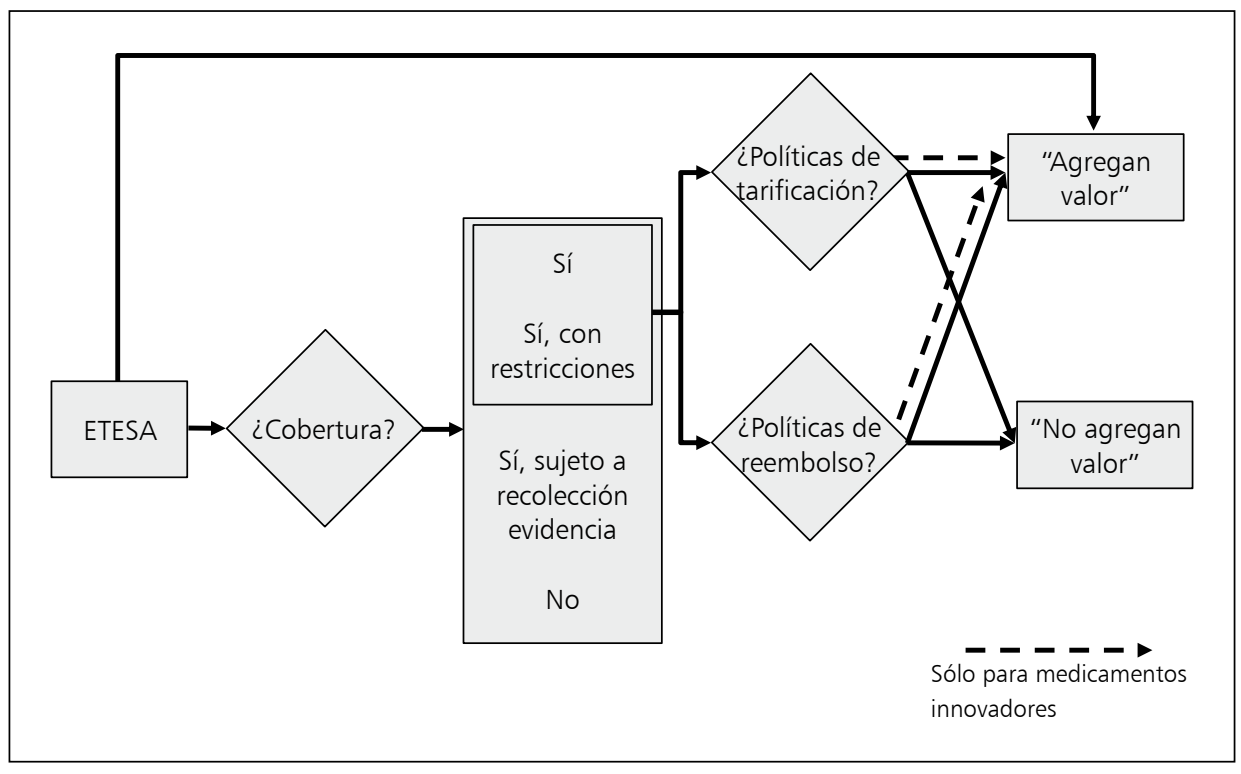


Una vez determinado el grupo de fármacos que serán cubiertos se llevan a cabo las denominadas "políticas de tarificación y reembolso" destinadas a influir en el precio de los mismos. Cabe destacar que los tratamientos que se someten a cobertura sujeto a recolección de evidencia sufren un proceso de negociación independiente y se mencionan en la última sección del documento.

El contexto institucional en el que se llevan a cabo estos procesos involucra habitualmente a distintas organizaciones. En algunos países la misma autoridad que tiene a cargo la decisión con respecto a cobertura se encarga de llevar a cabo las políticas de tarificación y reembolso, mientras que en otros esta decisión recae en distintas organizaciones. En tanto, los procesos de ETESA habitualmente están a cargo de organismos que intervienen mediante la entrega de recomendaciones, pero que no tienen injerencia directa sobre las decisiones mencionadas.

La relación entre ETESA y las políticas de tarificación y reembolso presenta importantes variaciones entre los distintos países. Siguiendo el esquema propuesto en la figura y de acuerdo a los planteado por otros autores ${ }^{6}$, se distinguen en este trabajo aquellas políticas que tienen dentro de sus objetivos evaluar la "agregación de valor" de los tratamientos, proceso que es llevado a cabo mediante ETESA, y a la vez se categorizan estas políticas dependiendo de si esta evaluación adopta formalmente o no un análisis de costo-efectividad. Cabe destacar que estos procesos no son excluyentes, tal es el caso de países que aplican políticas de tarificación diferenciales a los tratamientos innovadores con el objetivo de "agregar valor" mientras que aplican políticas de tarificación y reembolso tradicionales al resto de los fármacos (esto se representa mediante la línea segmentada en la Figura 1).

Siguiendo este esquema, la Tabla 1 propone una categorización para los distintos mecanismos de tarificación y reembolso basada en ambos criterios (agregación de valor y costo efectividad) sobre la cual se profundizará a continuación, incluyendo algunos ejemplos de países y agencias que aplican dichos mecanismos.

\section{Políticas de tarificación y reembolso que "no agregan valor"}

En este contexto se encuentran, entre otras, las políticas de negociación de precios por volumen, en que el precio se fija en relación a la cantidad transada; las de "precio techo o price caps", en que se fija un precio máximo; las de "costo + o cost plus", en que se fija un margen por sobre el costo; las de "comparación o referenciación interna o externa de precios o internal or external price referencing", donde se toman como referencia los precios de medicamentos similares en el mismo país o del mismo medicamento en un conjunto de países; y la regulación de la tasa de retorno, que no se considera una regulación directa, ya que no apunta a controlar el precio, pero que apunta a asegurar que no existan ganancias excesivas y al mismo tiempo recompensar la innovación ${ }^{7}$.

La referencia externa de precios es una política ampliamente utilizada, sin embargo, es a menudo criticada por descansar sobre criterios "arbitrarios" respecto, por ejemplo, del grupo de países con que se compara, el precio tomado para comparar, la frecuencia de revisión, etc., sin necesariamente adherir a un marco conceptual o teórico particular. En cualquier caso se reconoce que su racionalidad es clara, apuntando a que los países que la aplican no sobre-paguen por los mismos medicamentos en relación a sus países vecinos, y al mismo tiempo ejercer presión sobre los precios buscando eficiencia macroeconómica ${ }^{9}$.

Por otra parte, el esquema de precios de referencia determina el precio o límite de reembolso que el pagador cubrirá para cada uno de los productos de un grupo de referencia, mientras que el diferencial entre dicho precio y el precio efectivo del producto debe ser cubierto por el paciente. Los grupos de referencias pueden ser más acotados o más amplios según incluyan sólo medicamentos con el mismo principio activo, medicamentos con principios activos relacionados que son farmacológicamente equivalentes o productos cuyos principios activos no necesariamente están relacionados pero que tienen efectos terapéuticos comparables ${ }^{8}$.

\section{Políticas de tarificación y reembolso que "agregan valor"}

\section{Agregación de valor sin aplicar criterios de costo efectividad}

En los casos en que no se aplica formalmente este tipo de análisis, el objetivo es asegurar el financiamiento de medicamentos que entreguen 
Tabla 1. Categorización mecanismos de tarificación y reembolso

\begin{tabular}{|c|c|c|}
\hline \multicolumn{3}{|l|}{ Tarificación } \\
\hline \multicolumn{2}{|c|}{$\begin{array}{l}\text { Agrega Valor } \\
\text { Aplica formalmente criterios de Costo Efectividad }\end{array}$} & No agrega valor \\
\hline $\begin{array}{l}\text { - Negociación en base a costo efectividad: } \\
\text { formalmente se compara RICE vs umbral y } \\
\text { se intenta disminuir el precio del fármaco } \\
\text { para que se encuentra bajo el umbral. } \\
\text { - Ej. Reino Unido-NICE; Suecia-TLV; } \\
\text { Escocia-SMC } \\
\text { - Fijación de precio en base a costo efectivi- } \\
\text { dad: "Value Based Pricing" } \\
\text { - Ej. Reino Unido-NICE, a partir de } 2014\end{array}$ & $\begin{array}{l}\text { - Precio ligado a efectividad: se determina } \\
\text { si el medicamento entrega beneficios por } \\
\text { sobre la terapia actual, posteriormente se } \\
\text { negocia el precio o se permite que tenga } \\
\text { un precio mayor a otros referentes. } \\
\text { - Ej. Francia-HAS; Alemania-FJC-IQWiG } \\
\text { - Precio en base a innovación: se determina } \\
\text { si el medicamento es innovador y se le } \\
\text { permite en la negociación tener un precio } \\
\text { mayor. - Ej. Italia-AlFA (a partir de } 2013 \\
\text { se comienzan a introducir criterios de } \\
\text { costo efectividad) }\end{array}$ & $\begin{array}{l}\text { - PPRS: Reino Unido } \\
\text { - Tarificación en base a pre- } \\
\text { cios otros países (cuando } \\
\text { se utiliza como medida } \\
\text { aislada) } \\
\text { - Congelamiento de precios }\end{array}$ \\
\hline \multicolumn{3}{|l|}{ Reembolso } \\
\hline \multicolumn{2}{|c|}{$\begin{array}{l}\text { Agrega Valor } \\
\text { Aplica formalmente criterios de Costo Efectividad } \\
\text { Sí }\end{array}$} & No agrega valor \\
\hline $\begin{array}{l}\text { - Lista en base a costo efectividad: se inclu- } \\
\text { ye como medicamento a ser financiado } \\
\text { por el seguro si es que su RICE se encuen- } \\
\text { tra bajo el umbral. Puede restringirse a } \\
\text { subgrupos. } \\
\text { - Ej. Reino Unido-NICE; Suecia-TLV; } \\
\text { Escocia-SMC } \\
\text { - Acuerdos de riesgo compartido: si existe } \\
\text { incertidumbre con respecto a la costo } \\
\text { efectividad del tratamiento se financia } \\
\text { bajo condiciones específicas (investiga- } \\
\text { ción, resultados, otros). }\end{array}$ & $\begin{array}{l}\text { - Reembolso ligado a relevancia terapéuti- } \\
\text { ca: se clasifican en grupos y se entrega un } \\
\text { reembolso mayor a medicamentos con } \\
\text { relevancia terapéutica. } \\
\text { - Ej. Bélgica. }\end{array}$ & $\begin{array}{l}\text { - Listas que no incorporan } \\
\text { análisis de efectividad o } \\
\text { costo efectividad } \\
\text { - Grupos de referencia: se } \\
\text { asignan fármacos a gru- } \\
\text { pos terapéuticos y se fija } \\
\text { un reembolso máximo en } \\
\text { cada grupo. } \\
\text { - Ej. Alemania }\end{array}$ \\
\hline
\end{tabular}

Fuente: Elaboración propia.

beneficios terapéuticos al sistema de salud por sobre las alternativas existentes. Dos perspectivas que se enmarcan en este modelo son la evaluación según nivel de innovación y según efectividad.

Un ejemplo donde se adopta un mecanismo que evalúa el nivel de innovación es Italia, a través de la Agencia Italiana de Fármacos (AIFA). Bajo este modelo se pretende determinar el grado de innovación terapéutica en relación a la disponibilidad actual de tratamientos y a la eficacia demostrada por el medicamento en análisis, de tal forma que un fármaco de gran eficacia y destinado al tratamiento de una patología para la cual no existen alternativas de tratamiento será clasificado como de gran innovación terapéutica ${ }^{6}$.
Esta información se utiliza en la negociación con la industria para determinar el precio de un nuevo tratamiento.

Otra manera de evaluar la agregación de valor es mediante la efectividad del medicamento o su beneficio terapéutico. Un ejemplo de este mecanismo es Francia, en donde la Autoridad Nacional de Salud (HAS por sus siglas en francés) realiza evaluaciones de todos los tratamientos mediante dos fases: el Service Médical Rendu y el Amélioration du Service Médical Rendu'. La primera fase se encarga de determinar la efectividad terapéutica y en base a ello seleccionar los tratamientos que serán sujetos de cobertura y fijar el nivel de reembolso. La segunda fase compara el nuevo 
tratamiento con los actuales para determinar el precio. Si el tratamiento se demuestra más efectivo que sus comparadores se somete a un proceso de referencia externa. Si se demuestra que la efectividad relativa es limitada, se lleva a cabo un proceso de negociación con la industria. Si no demuestra mayor efectividad se someten a un esquema de precios de referencia.

Un proceso similar al anterior se lleva a cabo en Alemania con la participación del Instituto para la Calidad y Eficiencia en Salud (IQWiG por sus siglas en alemán) que se encarga de determinar si un nuevo tratamiento presenta un beneficio adicional en salud, caso en el cual se lleva a cabo una negociación con la industria para determinar el precio. Si se estima que no hay beneficio adicional, el tratamiento se somete a un esquema de precios de referencia ${ }^{10}$.

En todos estos ejemplos se utiliza ETESA como mecanismo para diferenciar aquellos medicamentos con mayor innovación terapéutica, efectividad o beneficio terapéutico de tal forma que estos se sometan a mecanismos de tarificación y reembolso que les permitan tener un precio mayor (y/o un mayor reembolso) en relación a otros alternativos.

\section{Agregación de valor aplicando criterios de costo efectividad}

En los casos en que el precio y/o reembolso es fijado en consideración a la costo-efectividad de los medicamentos innovadores dos elementos resultan fundamentales. Por una parte, la obtención de la razón incremental de costo-efectividad-RICE (en relación al medicamento comparador); y por otra, el umbral de disposición a pagar por unidad de beneficio adicional en salud definido para el país bajo análisis, esto ya sea a nivel general considerando a todos los individuos afectados por la condición, o sólo a un sub-grupo de éstos que se beneficiarían de mayor manera con el medicamento. Lo que se requiere es que la RICE sea menor al umbral definido y, por lo tanto, el precio del medicamento debe ser menor a aquel precio que haga los tratamientos equivalentes, asegurando que las ganancias en salud esperadas sean al menos iguales a los costos adicionales asociados al medicamento (i.e. beneficio neto igual a cero), lo cual se puede lograr a través de negociaciones entre el regulador y la industria.
En este contexto, la tarificación basada en valor (value-based pricing o VBP) es un esquema de fijación del precio de los medicamentos para los distintos sub-grupos de pacientes basándose directamente en su costo-efectividad, en que el precio ofrecido al productor es mayor para aquellos grupos de pacientes para los que el medicamento resulta más costo-efectivo, y menor para aquellos en que resulta menos costo-efectivo (i.e. menú de precios), permitiendo que las ganancias en salud puedan superar a los costos (i.e. beneficio neto positivo $)^{9}$. De esta forma, bajo los principios del VBP se espera que en el corto plazo los medicamentos sean aprobados para uso sólo si los beneficios en salud que entregan superan a sus costos de oportunidad, mientras que en el largo plazo los incentivos estarían dados para que la industria desarrolle tecnologías tendientes a agregar valor y ser costo efectivos ${ }^{10}$.

En escenarios de aplicación ex-ante de VBP, en que no existe información suficiente para el desarrollo del análisis de costo-efectividad (e.g. incertidumbre respecto de la efectividad del medicamento), se pueden considerar esquemas de "riesgos compartidos" (risk sharing) entre la industria y el financiador, o también denominados "esquemas de acceso a pacientes" (patient access schemes) ${ }^{6}$. En estos casos la cobertura del medicamento se condiciona a los resultados obtenidos luego que la nueva evidencia se encuentre disponible, por ejemplo, luego de que es aprobado para su uso ${ }^{11}$.

\section{Conclusiones}

Las políticas de tarificación y reembolso de medicamentos tienen como uno de sus objetivos el asegurar que los precios pagados, ya sea por los individuos o los seguros, sean "razonables" y, por lo tanto, la población pueda acceder a éstos. La incorporación de ETESA en este proceso permite además asegurar que los sistemas financien medicamentos que agregan valor y orientar los esfuerzos de la industria hacia el desarrollo de este tipo de terapias. En un contexto como el chileno, con un alto gasto de bolsillo por parte de los hogares y del cual la proporción más importante corresponde a medicamentos ${ }^{12}$, el avanzar hacia una política de medicamentos que incluya criterios de tarificación y reembolso, y en que los 
seguros jueguen un rol más protagónico resulta fundamental.

La experiencia internacional es heterogénea en su diseño y, pese a que existen similitudes en los objetivos y metodologías entre algunos países que permiten categorizar ciertos modelos, la institucionalidad e implementación de este tipo de políticas depende en gran medida de las características de los sistemas de salud en los cuales se insertan.

Algunas consideraciones importantes en relación a mecanismos asociados a la costoefectividad, y en particular al "value-based pricing" dicen relación con la necesidad de contar con un umbral definido para establecer la disposición a pagar por los outcomes entregados por tratamiento asociado al medicamento. Esto a su vez requiere como tema más amplio la institucionalización de la ETESA para la toma de decisiones.

Por otra parte, para implementar las metodologías necesarias para fijar precios asociados al valor se requiere contar con una cantidad importante de información contexto-específica (uso de QALYs, efectividades para distintos subgrupos, etc.), además de las capacidades para llevar a cabo los análisis.

En conclusión, existe una variada gama de políticas de tarificación y reembolso que han sido implementadas en distintos países del mundo. Por otro lado, Chile enfrenta el desafío de modernizar su política de acceso a tecnologías sanitarias. La revisión detallada de estas experiencias puede dar importantes luces de eventuales sistemas de cobertura, tarificación y reembolso que puedan ser implementados en nuestro país.

\section{Referencias}

1. Mrazek M, Frank R. The Off-patent Pharmaceutical Market. Capitulo14 en Regulating Pharmaceuticals in Europe: Striving for efficiency, equity and quality. Editado por E. Mossialos, M. Mrazek and T. Walley. Open University
Press: Buckingham; 2004.

2. Mossialos E, Walley T, Mrazek M. Regulating pharmaceuticals in Europe: an overview. Capitulo 1 en Regulating Pharmaceuticals in Europe: striving for efficiency, equity and quality. Editado por Mossialos E, Mrazek M, Walley T. Open University Press: Buckingham; 2004.

3. Mrazek M, Mossialos E. Regulating Pharmaceutical Prices in the European Union. Capitulo 6 en Regulating Pharmaceuticals in Europe: Striving for efficiency, equity and quality. Editado por Mossialos E, Mrazek M, Walley T. Open University Press: Buckingham; 2004.

4. McGuire A, Drummond M, Rutten F. Reimbursement of Pharmaceuticals in the European Union. Capítulo 7 en Regulating Pharmaceuticals in Europe: striving for efficiency, equity and quality. Editado por Mossialos E, Mrazek M, Walley T. Open University Press: Buckingham; 2004.

5. Stafinski T, Menon D, Davis C. Role of centralized review processes for making reimbursement decisions on new health technologies in Europe. Clinicoecon Outcomes Res 2011; 3: 117-86.

6. Kanavos P, Manning J, Taylor D, Schurer W, Checchi, $\mathrm{K}$. Implementing value-based pricing for pharmaceuticals in the UK. 2020 Health report; 2010.

7. Kanavos P. Overview of pharmaceutical pricing and reimbursement regulation. LSE Health, London, U.K; 2001.

8. Lopez-Casasnovas G, Puig-Junoy J. Review of the literature on reference pricing. Health Policy 2000; 54 (2): 87-123.

9. Kanavos P, Nicod E, Espin J, van den Aardweg. Shortand Long- Term Effects of Value-Based Pricing vs. External Price Referencing. EMINET; 2010.

10. Ognyanova D, Zentner A, Busse, R. Pharmaceutical reform 2010 in Germany: striking a balance between innovation and affordability. Eurohealth 2011; 17 (1): 11-3.

11. Claxton K, Briggs A, Buxton M, Culyer A, McCabe C, Walker S, et al. Value based pricing for NHS drugs: an opportunity not to be missed?. BMJ 2007; 336: 251-4.

12. Cid C, Prieto L. El gasto de bolsillo: el caso de Chile 1997 y 2007. Rev Panam Salud Pública 2007; 31 (4): 310-6. 